\title{
Tool for Osteoarthritis Risk Prediction (TOARP) over 8 years using Baseline Clinical Data, X-ray, and MR imaging - Data from the Osteoarthritis Initiative
}

\author{
Gabby B. Joseph, PhD'1, Charles E. McCulloch, PhD $^{2}$, Michael C. Nevitt, PhD $^{2}$, Jan \\ Neumann, MD11, Alexandra S. Gersing, MD1, Martin Kretzschmar, MD1, Benedikt J. \\ Schwaiger, MD ${ }^{1}$, John A Lynch, PhD $^{2}$, Ursula Heilmeier, MD $^{1}$, Nancy E. Lane, MD $^{3}$, and \\ Thomas M. Link, MD, PhD ${ }^{1}$ \\ ${ }^{1}$ Department of Radiology and Biomedical Imaging, University of California, San Francisco \\ ${ }^{2}$ Department of Epidemiology and Biostatistics, University of California, San Francisco \\ ${ }^{3}$ Department of Rheumatology, University of California, Davis
}

\begin{abstract}
Background-Osteoarthritis, a multifactorial disease causing joint degeneration, often leads to severe disability. The rising rates of disability highlight the need for implementing preventative measures at early stages of disease, which would especially benefit subjects at high risk for OA development.
\end{abstract}

Purpose-To develop a risk prediction tool for moderate-severe Osteoarthritis (TOARP) over 8 years based on subject characteristics, knee radiographs, and MRI data at baseline using data from the Osteoarthritis Initiative (OAI).

Study Type-Retrospective

Subjects-641 subjects with no/mild radiographic OA (KL 0-2) and no clinically significant symptoms (WOMAC 0-1) were selected from the OAI.

Field Strength/Sequence-MR images were obtained using 3.0 Tesla (Siemens Magnetom Trio, Erlangen, Germany).

Assessment-Compartment-specific cartilage and meniscus morphology and cartilage $\mathrm{T} 2$ were assessed. Baseline subject demographics, risk factors, KL score, cartilage WORMS score, presence of meniscus tear, and cartilage $\mathrm{T} 2$ were used to predict the development of moderate/ severe $\mathrm{OA}(\mathrm{KL}=3-4$ or WOMAC pain $\geq 5$ or TKR) over 8 years.

Statistical Tests-Best subsets variable selection followed by cross-validation were used to assess which combinations of variables best predict moderate/severe OA.

Results-Model 1 included KL score, previous knee injury in the last 12 months, age, gender, and BMI. Model 2 included all variables in Model 1 plus presence of cartilage defects in the

Corresponding author: Gabby B. Joseph PhD, Department of Radiology and Biomedical Imaging, University of California, San Francisco, 185 Berry St, Suite 350, San Francisco, CA 94158, gabby.joseph@ucsf.edu, Phone: 415.353.4566, Fax: 415.476.0616. 
lateral femur and patella, and presence of a meniscal tear. Model 3 included all variables in Models 1 and 2, plus cartilage T2 in the medial tibia and medial femur. Compared to Model 1 (cross-validated AUC $=0.67$ ), Model 3 performed significantly better $(\mathrm{AUC}=0.72, \mathrm{p}=0.04$ ) while Model 2 showed a statistical trend $(\mathrm{AUC}=0.71, \mathrm{p}=0.08)$.

Data Conclusion-We have established a risk calculator for the development of moderate/ severe knee OA over 8 years that includes radiographic and MRI data. The inclusion of MRIbased morphological abnormalities and cartilage $\mathrm{T} 2$ significantly improved model performance.

\section{Keywords}

Osteoarthritis; Cartilage T2; MRI; risk model

\section{INTRODUCTION}

Osteoarthritis (OA), a multifactorial disease that causes joint degeneration, affects 14 million U.S. adults, and often leads to severe disability (1) and total knee replacement (TKR). The rising rates of TKR(2) and secondary revision surgeries(3) highlight the need for implementing preventative measures such as lifestyle modifications, at early stages of disease. Such preventative measures would be particularly beneficial for subjects at high risk for OA development.

In addition to the known clinical risk factors for OA (including obesity and previous injury(4)), imaging of the knee joint may assist in the identification of subjects at high risk. Kellgren-Lawrence (KL) knee radiographic scores are positively associated with knee pain(5), and varus knee alignment at baseline is associated with a 4-fold increase in medial OA progression(6). In addition, cartilage damage and meniscal tears as assessed with MRI, have been shown associated with incident radiographic knee OA 2-4 years later (7). More recently, MRI T2 mapping, which identifies biochemical changes in cartilage including abnormalities of collagen fiber orientation(8), has been shown to predict radiographic and symptomatic knee OA(9). MRI T2 measures early degenerative changes in knee cartilage that occur prior to macroscopic cartilage defects and thinning. Thus, a composite model consisting of clinical risk factors, and imaging data may help identify subjects at high risk for OA.

Clinical and imaging prediction tools are used by clinicians to identify patients who might benefit from an intervention, either medical or surgical, to prevent an outcome. One such model, the WHO fracture risk assessment tool (FRAX), is used to identify subjects at risk of hip and major osteoporotic fractures using clinical risk factors (including age, sex, BMI, prior fracture and parental history of hip fracture) and a bone density measurement, developed with Poisson regression (10)). Similarly, this study aims to develop a risk prediction tool for moderate/severe OA. The purpose of this study was to develop a Tool for Osteoarthritis Risk Prediction (TOARP) over 8 years based on subject characteristics, knee radiographs, and MRI data at baseline using data from the Osteoarthritis Initiative (OAI). 


\section{MATERIALS AND METHODS}

\section{Subject Selection}

This study utilizes data from the Osteoarthritis Initiative (OAI; http://www.oai.ucsf.edu/) (11), a multi-center, longitudinal study of persons aged 45-79 years at enrollment, aimed at assessing biomarkers in OA including those derived from MR imaging. The OAI dataset includes both MRI and radiographic images of subjects scanned over eight years. This database can be used to evaluate MRI biomarkers for the development and progression of OA. The study protocol, amendments, and informed consent documentation were reviewed and approved by the local institutional review boards of all participating centers.

Participants for the present study were selected from the OAI, which excluded individuals with inflammatory arthropathies (i.e. rheumatoid arthritis), MRI contraindications, positive pregnancy test, bilateral total knee joint replacement, and co-morbid conditions that may affect the ability to participate in the study. Specific inclusion criteria for the present study were a baseline radiographic Kellgren Lawrence score $(\mathrm{KL}) \mathcal{\Omega}$ in the right knee and no symptoms in the right knee (Western Ontario and McMaster Universities Arthritis Index (WOMAC) of 0/1). A WOMAC pain score threshold of $\leq$ was chosen based on a previous study(12) that reported a minimal perceptible change in pain required a $10 \%$ difference [using the VAS scale], which equates to a change greater than 2 utilizing the WOMAC Likert scale. The sample of knees was selected from MRI scans that had both T2 and WORMS score assessments in the right knee(13-17), and also had complete data for known knee OA risk factors including family history of knee replacement and previous injury. The prior studies analyzing T2/WORMS had vastly different goals from the current study. There are no selection biases since the distribution of subject characteristics in the current study is similar to that of the OAI database. Exclusion criteria included baseline knee deformity of the knee joint, total joint replacements in the right knee, MRI evidence of subchondral or stress fractures of the knee or abnormalities that did not fit into the spectrum of OA and indicated other severe disease, such as tumor or inflammation. Based on our inclusion and exclusion criteria right knees from 641 participants were selected and analyzed, Figure 1.

\section{MR Imaging}

MR images were obtained using four identical 3.0 Tesla (Siemens Magnetom Trio, Erlangen, Germany) scanners in Columbus, Ohio; Baltimore, Maryland; Pittsburgh, Pennsylvania; Pawtucket, Rhode Island. The following sequences were acquired: sagittal 2D intermediate-weighted fast spin-echo sequence (TR/TE $=3200 / 30 \mathrm{~ms}$, spatial resolution $=0.357 \mathrm{mmx} 0.511 \mathrm{~mm}$, slice thickness $=3.0 \mathrm{~mm}$ ), coronal $2 \mathrm{D}$ proton density fast spin-echo sequence $(\mathrm{TR} / \mathrm{TE}=3700 / 29 \mathrm{~ms}$, spatial resolution $=0.365 \mathrm{mmx} 0.456 \mathrm{~mm}$, slice thickness $=3.0 \mathrm{~mm})$, and sagittal 3D dual-echo in steady state sequence $(\mathrm{TR} / \mathrm{TE}=16.3 / 4.7 \mathrm{~ms}$, spatial resolution $=0.365 \mathrm{mmx} 0.456 \mathrm{~mm}$, slice thickness $=0.7 \mathrm{~mm}$ ). A sagittal $2 \mathrm{D}$ multi-slice multi-echo sequence (MSME, TR=2700ms, $\mathrm{TE}_{1}-\mathrm{TE}_{7}=10-70 \mathrm{~ms}$, spatial resolution $=0.313 \mathrm{~mm} \times 0.446 \mathrm{~mm}$, slice thickness $=3.0 \mathrm{~mm}$, and $0.5 \mathrm{~mm}$ gap) was used for cartilage $\mathrm{T} 2$ measurements(18). 


\section{Image Analysis}

Radiography-based KL grade and Knee Alignment-Baseline and annual radiographic KL grades(19) over 8 years were provided in the OAI dataset. Subjects with baseline $\mathrm{KL}$ grades of $0-2$ were selected, and worsening was defined as developing a KL grade of 3-4 over 8 years. Baseline knee alignment (femur-tibia angle) was measured based on a method developed by Iranpour-Boroujen et al (20). This method had high reproducibility with an ICC of intra- and inter-reader reproducibility of 0.96 and 0.98 respectively(20).

WORMS Scoring-MR images of the right knee obtained at the baseline visit were reviewed on picture archiving communication system (PACS) workstations (Agfa, Ridgefield Park, NJ, USA). Three radiologists with 7-, 5- and 5-years of experience graded cartilage lesions. In equivocal cases, a consensus reading was performed with a musculoskeletal radiologist with 23-years of experience. Baseline cartilage lesions were assessed in five regions (patella, medial femur, medial tibia, lateral femur and lateral tibia) using a modified semi-quantitative whole-organ magnetic resonance imaging score (WORMS) (21). The highest score of any lesion was recorded for each region. For calibration purposes, 20 cases were read simultaneously by the four readers in consensus. A binary variable representing a cartilage defect was defined as positive if WORMS grade was $\geq 2$ in (1) each region individually (patella, medial femur, medial tibia, lateral femur and lateral tibia) and (2) in any region overall. Meniscal lesions were graded separately in 6 regions (medial/lateral and anterior/body/posterior) using the following 4-point scale: 0normal; 1-intrasubstance signal; 2-non-displaced tear; 3-displaced or complex tear; 4complete destruction/maceration. A binary variable representing a meniscus tear was defined as positive if WORMS grade was $\geq 2$ in any meniscus region.

T2 measurements-All baseline images were analyzed using a Sun Workstation (Sun Microsystems, Palo Alto, CA, USA). Semi-automatic cartilage segmentation of lateral/ medial femur, lateral/medial tibia, and patella regions was performed as previously described, using an in-house, spline-based software based on MATLAB (MathWorks, Natick, Massachusetts)(22). Our segmentations covered cartilage slices that did not contain partial volume effects; we also excluded sections with any compromised image quality such as artifacts.

Validated methods for obtaining a T2 map of the cartilage have been published $(22,23)$. T2 maps were computed from the MSME images on a pixel-by-pixel basis using 6 echoes ( $\mathrm{TE}=20-70 \mathrm{~ms}$ ) and 3 parameter fittings accounting for noise $(24,25)$, and averaged over all of the slices in each cartilage region, accounting for the number of pixels in each slice. The first echo ( $\mathrm{TE}=10 \mathrm{~ms}$ ) was not included in the $\mathrm{T} 2$ fitting procedure in order to reduce potential errors resulting from stimulated echoes, and a noise-corrected algorithm was implemented(24,25). The cartilage $\mathrm{T} 2$ reproducibility results have been described previously $(22,23)$. The intra-reader reproducibility of mean $\mathrm{T} 2$ was determined by segmenting the cartilage in 15 subjects, three times by one operator. The inter-reader reproducibility was determined by segmenting five subjects, three times each by two operators. The mean T2 values had root mean square (RMS) coefficients of variation (CV) 
ranging from $0.83 \%$ in the medial femur to $3.21 \%$ in the patella for intra-reader reproducibility, and from $1.22 \%$ in the patella to $1.86 \%$ in the lateral tibia for inter-reader reproducibility.

\section{Statistical Analysis}

Statistical analysis was performed using STATA version 13 software (StataCorp LP, College Station, TX). The outcome variable was development of moderate to severe radiographic or symptomatic knee OA, defined any of the following during up to 8 years of follow-up: worsening to KL grade 3-4 OA, a WOMAC pain score of $\geq 5$ at any two follow-up timepoints, or an incident total knee replacement (TKR) in the right knee, confirmed by medical records and/or knee radiographs. We included KL score as an outcome for this study, as radiographic OA is considered a standard outcome definition for OA (26). The predictors for this study were: subject characteristics (age, gender, and BMI, locked to remain in the model), baseline risk factors, KL score, knee alignment, presence of cartilage defect, presence of meniscus tear, and cartilage T2 as listed in Figure 2. Logistic regression was used for analysis to be consistent with the published literature on OA. A STATA algorithm(27) which utilized "leaps-and-bounds(28) was used to perform best subsets variable selection with logistic regression to assess which combinations of the above-listed variables best predicted moderate to severe radiographic OA (outcome). Akaike's information criteria (AIC) for each combination of predictors (the lowest being the most desirable) were assessed, to compare each model's goodness of fit relative to one another. Next, 10-fold cross validation was performed for the models with the lowest AICs (within 10)(29), and the Discrimination Index (DI, defined as the average of the predicted probability of an event among the individuals with an event minus the average of the predicted probability of an event among individuals without an event) was quantified (30). The discrimination index defines the percentage differences in risk between the subjects who developed a positive outcome compared to a negative outcome. We did not use a training and testing dataset in the study due to the small sample size with low outcome percentage (12.48\%). Thus, we chose 10-fold cross-validation(31).

First, the analysis was performed for a base model, which included clinical data only (age, gender, and BMI). Next, the analyses were performed three times independently to develop 3 models: (Model 1) being the least sophisticated and included base model and radiography, (Model 2) being more sophisticated and included the base model, radiography, and MRI WORMS scoring, and (Model 3) being the most sophisticated and included the base model, radiography, MRI WORMS scoring, and MRI T2 (Figure 2). Ten-fold cross-validated receiver operating characteristic (ROC) analyses were used to obtain an unbiased assessment of each model; then cross validated area under the curves (AUCs) were compared using the test of DeLong, DeLong and Clarke-Pearson(32).

\section{RESULTS}

\section{Subject Characteristics}

The 641 participants in this study had a mean age of $56.4 \pm 7.5$ years and a mean BMI of $27.0 \pm 4.3 \mathrm{~kg} / \mathrm{m}^{2}$ at baseline. The distribution of KL grades and other participant 
characteristics are listed in Table 1. 80 subjects (12.48\%) had a positive outcome (either an incident TKR [ $n=8,1.25 \%$ ], worsening to KL 3 or 4 [n=34, 5.31\%], or progression to a WOMAC pain score of $\geq 5$ [n=53, 8.27\%]). Three hundred and eighty-one subjects (59.44\%) had cartilage defects in any cartilage region while 190(29.64\%) had a meniscus tear in any meniscus region.

\section{OA Risk Models}

The following models best predicted the development of knee OA over 8 years (Table 2): The Base model included age, gender and BMI. Model 1 added the radiography-based KL score, knee alignment, and previous knee injury in the last 12 months (cross-validated $\mathrm{DI}=0.048$ ). Model 2 included all variables in Model 1 plus presence of cartilage defects in the lateral femur and patella, and presence of a meniscal tear (cross validated DI $=0.084$ ). Model 3 included all variables in Model 1 and 2, plus mean cartilage T2 in the medial tibia and medial femur (cross validated DI=0.11). Compared to Model 1 with a cross-validated area under the ROC curve (AUC) $=0.67$, Model 3 performed significantly better $(\mathrm{AUC}=0.72$, $\mathrm{p}=0.04$ ) while Model 2 showed a statistical trend $(\mathrm{AUC}=0.71, \mathrm{p}=0.08)$. However, there was no difference in performance between Models 2 and 3. All models had significantly $(\mathrm{p}<0.05)$ greater AUCs compared to a base model consisting of age, gender, and BMI, Figure 3, demonstrating the added value of imaging for risk prediction. These results demonstrate that including both cartilage T2 and WORMS significantly improves model performance compared to a model with risk factors and KL-score alone. The AUC results were similar after a sensitivity analysis excluding African American subjects ( $\mathrm{n}=56,8 \%$, AUC for Model $3=0.74$ ). Figure 4 illustrates the improvement of model risk stratification from Model 1 to Model 3, especially in subjects that develop OA over 8 years.

\section{Tool for Individualized OA Risk Prediction (TOARP)}

Figure 5a illustrates a risk calculator graphic designed for use in the clinic; Figure 5b illustrates the isolated effects of low, medium, and high medial femur cartilage T2 (from the T2 distribution in this study) on OA risk probability, while keeping the other subject characteristics (risk factors, KL score, and WORMS scores) constant. For example, a 69 year old female with a BMI of $25.8 \mathrm{~kg} / \mathrm{m}^{2}$, a previous injury, KL score of 2, lateral femur cartilage defect, medial meniscus tear, and a medial femur cartilage T2 of 43ms ( $~ 98^{\text {th }}$ percentile based on a reference database of subjects without cartilage degeneration(33)) would have $\sim 75 \%$ risk for progression of OA development, while a female with the same characteristics and a medial femur cartilage $\mathrm{T} 2$ of $31 \mathrm{~ms}\left(\sim 2^{\text {nd }}\right.$ percentile $)$ would have a risk of $\sim 34 \%$.

\section{DISCUSSION}

This study created a composite subject-specific risk assessment model for OA development over 8 years that includes clinical and advanced MR imaging data. The three models established in this study range from least sophisticated (including subject characteristics, risk factors and radiography) to most sophisticated with WORMS and cartilage T2. Compared to the least sophisticated model, the addition of MR imaging parameters increased the AUC values, demonstrating significance for the model with WORMS and T2 
and a statistical trend for WORMS alone. Overall, the three models provide versatility for OA risk prediction and they could be used by clinicians to provide individualized assessments to patients, and could motivate lifestyle changes to lower risk of OA progression.

While previous studies have developed risk prediction models for OA(34-37), our study is different as it includes MR imaging and assessment of known risk factors for OA development over 8 years in subjects without, or with only mild, radiographic OA and no symptoms of OA at baseline. A variety of OA risk calculators have been developed which range in complexity: Some included only subject demographics, clinical factors, and risk factors without imaging(34), while others integrate biochemical markers and radiographybased KL scores in their OA prediction model(35). Kerkhoff et al.(35) reported similar accuracy, when including clinical variables in addition to genetic scores and biochemical markers (AUC $\sim 0.66$, outcomes spanning 4 to 10 years); however the inclusion of baseline radiographic KL score increased the AUC to 0.79 , demonstrating the importance of imaging for risk prediction. The different AUCs in this study compared to the current study may be due to the differences in the subject inclusion criteria (Kerkoff et al. included subjects with KL 0-1 while we included KL 0-2) and outcome definitions (Kerkoff et al. used incident $\mathrm{OA}$ defined by $\mathrm{KL} \geq 2$ while our outcome was composite). In addition to KL score, we further investigated the role of advanced MR imaging morphology and T2 values (indicative of cartilage extracellular matrix (ECM) composition) for OA prediction, and demonstrated that the addition of these advanced imaging techniques improves model discrimination.

Two key features of the models developed in this study are 1) individualized assessments and 2) inclusion of advanced MR imaging. Individualized risk profiles are essential for developing personalized prevention strategies for OA, and for motivating subjects to adhere to recommendations. In addition to subject characteristics, individualized assessments that incorporate advanced MR imaging allow clinicians to consider joint morphology and cartilage biochemical composition when formulating their treatment plans. While the model with radiography findings alone already provides fair prediction of OA risk probability, cartilage T2 relaxation time measurements improved prediction. Studies have shown that cartilage T2 can predict morphologic OA development (with outcomes of radiographic OA and changes in cartilage morphology) and symptomatic progression, highlighting the importance of T2 as a risk factor for OA development $(9,23,38)$. Given that cartilage T2 can detect the earliest stages of cartilage ECM degeneration prior to irreversible cartilage defects, $\mathrm{T} 2$ is a distinctive feature of our risk prediction, and the novelty of this study stems from the development of a model that incorporates both standard radiographic assessment and MR imaging. Thus, an individualized risk assessment that includes cartilage T2 may be used to identify subjects at high risk for the development of OA but at early stages of cartilage biochemical degeneration, at which point preventative efforts may be most effective.

The model that included lateral femur and patella cartilage lesions had the best performance possibly due to the fact that lateral femur (18\%) and patella lesions (66\%) were found most prevalent out of all cartilage compartments in subjects with risk factors for OA(39). The medial femur and tibia T2 had the best model performance compared to other T2 
compartments. Several reasons could account for this: medial OA occurs more frequently than lateral OA $(40,41)$, data from the OAI show that decreases in cartilage thickness over one year were greater in the medial than the lateral compartment (42), meniscus and cartilage lesions are more prevalent on the medial side of the joint (41), and the medial femur is a concentrated region of weight-bearing (41). Also, medial femur and tibia T2 has been shown associated with progression of OA (4).

We performed a sensitivity analysis examining the performance of the models excluding subjects with $\mathrm{KL}=2$. Out of the 519 subjects with $\mathrm{KL}=0$ or 1 , only 48 had a positive outcome. In the models excluding $\mathrm{KL}=2$, the cross validated AUC of the model with $\mathrm{T} 2$ was 0.67 and the model with radiography was 0.62 . These results may be affected by the lower sample size and the lower occurrence of a positive outcome in the subset of subjects with $\mathrm{KL}<2$. Also, since $\mathrm{KL}$ grade is an important predictor in the models, restricting the range to KL 0/1 will worsen the ability to risk stratify, and thus the AUC will be reduced. However, the positive aspect of including KL 2 is that it improves the prediction ability of the models.

Preventative efforts such as weight reduction $(17,43)$ and various levels of exercise $(16,44)$ may decrease risk for OA progression and may be advised after assessing an individual's long term risk for OA. One study found that a weight loss of 5\% body weight over 30 months decreased the risk of incident radiographic knee OA(45); another study suggested that moderate exercise may be a "good treatment" for subjects at high risk for OA(46), and a meta-analysis showed that long term weight loss is increased when diet and physical activity are combined(47). Weight loss also improves joint health and is associated with reduced medial cartilage volume (48), and with improvement in the cartilage quality (increase proteoglycan content) and reduced thickness in the medial cartilage(49). Thus, BMI is a modifyable risk factor for OA, and weight loss could be recommended if a subject is obese and at high risk for OA. In addition, subjects that play sports may benefit from injury prevention programs that have been shown to decrease the rate of injury $(50,51)$, and consequently decrease the rate of incident $\mathrm{OA}$. Thus, the risk prediction models developed in this study could motivate individuals to adhere to tailored disease modification strategies, and consequently decrease their risk for OA.

While model performance was significantly improved when comparing Model 1 (radiography) vs. Model 3 (radiography+WORMS+T2), Model 2, which included radiography+WORMS, was not significantly different from Model 3. Based on their AUCs, models 2 and 3 and were characterized as having "fair" performance; however similar performance values were found for the FRAX score (52). Overall, in a clinical environment, a model with MR-based WORMS may be sufficient if T2 is not available. However, if T2 is available, the additional information could aid in risk stratification by providing information on early biochemical cartilage changes, which cannot be detected using WORMS or radiographic findings. A model that includes T2 may be particularly beneficial for research trials targeting therapeutic interventions for early stages of disease. It should be noted that efforts are in place to standardize T2 mapping through the Quantitative Imaging Biomarker Alliance and automatic segmentation algorithm are developed $(53,54)$. Based on these developments it is likely that reproducible techniques and automated analysis algorithms are 
available in the near future. In the meantime two alternative models are available which are clinically applicable using standard imaging technologies.

Several limitations are pertinent to this study including the use of a composite outcome, inclusion of only cartilage $\mathrm{T} 2$ and no other compositional measures, the challenges and costs to obtain standardized MR imaging and perform T2/WORMS analysis, and lack of external validation in other cohorts. While singular outcomes would have been ideal, a composite outcome was chosen to obtain a broader clinical significance/applicability. In addition to cartilage $\mathrm{T} 2$, it would be beneficial to study other quantitative cartilage assessments such as T1rho mapping, however only $\mathrm{T} 2$ was available in the OAI. We did not specifically assess chondrocalcinosis (CPPD) in this cohort, but we did look at this in a different study where we analyzed 2122 subjects and found CPPD only in 99 subjects (4.7\%)(55), given the small number of subjects with CPPD we do not expect a significant impact of CPPD in this relatively young cohort with no or only mild degenerative changes. Bone marrow edema pattern and effusion were not included which may be considered as another limitation, however, these are less frequent in early stages of degenerative joint disease and are not stable (often appearing and resolving). Thus, we decided not to include them in this model. We did not assess the secondary knee in the models due to the fact that cartilage $\mathrm{T} 2$ was only available in the right knee in the OAI. Thus, we did not account for potential between-knee interactions such as malalignment in the secondary knee (in this cohort only $n=23$ subjects had contralateral $\mathrm{KL}>=3$ at baseline), which may have altered loading patterns in the primary knee, or knee injury in the secondary knee. In addition, we were unable to perform external validation as, to the best of our knowledge, no large longitudinal databases with cartilage T2 exist. Also, Since T2 values are known to vary based on acquisition methods, vendors, coils and post-processing techniques, a standardized imaging protocol would be necessary for these models to be utilized clinically. In addition, implementing a model with cartilage T2 may be complicated in the clinic due the required cartilage segmentation and post processing, which require a significant amount of manpower and time. However, we believe that the ongoing work to standardize T2 mapping through the Quantitative Imaging Biomarker Alliance and to implement automatic segmentation techniques using Artificial Intelligence Algorithms will facilitate the translation of cartilage T2 mapping clinically. Currently a model including WORMS is clinically more feasible as MRI sequences are routinely acquired, and a radiologist can detect the presence of focal cartilage lesions or meniscal tears without difficulty though reproducibility may vary. Other concerns that may be raised are, that radiography and clinical data are not always routinely collected and may therefore be challenging to implement in a risk prediction model; standardized questionnaires and patient management, however, would facilitate these issues. Despite these limitations, we believe this study is the first step in the development of a risk prediction model that includes advanced MR imaging.

In conclusion, this study showed that a risk prediction model that includes advanced MR imaging has a higher discrimination than a model with only subject demographics, risk factors, and radiography. Since the difference between models 1 and 3 reached statistical significance and the difference between 1 and 2 did not, perhaps a larger study should be undertaken to assess if model 2 is sufficient for risk prediction. Overall, information about an individual's risk for OA would be critical for the development of personalized treatment 
plans and preventative lifestyle interventions such as weight loss or exercise modification to improve long-term symptoms and overall knee degeneration.

\section{Supplementary Material}

Refer to Web version on PubMed Central for supplementary material.

\section{Acknowledgments}

\section{Role of Funding Source:}

The OAI is a public-private partnership comprised of five contracts (N01-AR-2-2258; N01-AR-2-2259; N01AR-2-2260; N01-AR-2-2261; N01-AR-2-2262) funded by the National Institutes of Health, a branch of the Department of Health and Human Services, and conducted by the OAI Study Investigators. Private funding partners include Merck Research Laboratories; Novartis Pharmaceuticals Corporation, GlaxoSmithKline; and Pfizer, Inc. Private sector funding for the OAI is managed by the Foundation for the National Institutes of Health.

Grant Support: This study was funded by NIH P50-AR060752 and NIH R01-AR064771

\section{References}

1. Deshpande BR, Katz JN, Solomon DH, et al. Number of Persons With Symptomatic Knee Osteoarthritis in the US: Impact of Race and Ethnicity, Age, Sex, and Obesity. Arthritis care \& research. 2016; 68(12):1743-1750. [PubMed: 27014966]

2. Carr AJ, Robertsson O, Graves S, et al. Knee replacement. Lancet. 2012; 379(9823):1331-1340. [PubMed: 22398175]

3. Center ME-bP. Services UDoHaH. Evidence Report/Technology Assessment. Vol. 86. Minneapolis: Agency for Healthcare and Research Quality; 2003. Total Knee Replacement.

4. Zhang Y, Jordan JM. Epidemiology of osteoarthritis. Clinics in geriatric medicine. 2010; 26(3):355369. [PubMed: 20699159]

5. Neogi T, Felson D, Niu J, et al. Association between radiographic features of knee osteoarthritis and pain: results from two cohort studies. Bmj. 2009; 339:b2844. [PubMed: 19700505]

6. Sharma L, Song J, Felson DT, Cahue S, Shamiyeh E, Dunlop DD. The role of knee alignment in disease progression and functional decline in knee osteoarthritis. Jama. 2001; 286(2):188-195. [PubMed: 11448282]

7. Sharma L, Nevitt M, Hochberg M, et al. Clinical significance of worsening versus stable preradiographic MRI lesions in a cohort study of persons at higher risk for knee osteoarthritis. Ann Rheum Dis. 2016; 75(9):1630-1636. [PubMed: 26467570]

8. Xia Y. Magic-angle effect in magnetic resonance imaging of articular cartilage: a review. Invest Radiol. 2000; 35(10):602-621. [PubMed: 11041155]

9. Liebl H, Joseph G, Nevitt MC, et al. Early T2 changes predict onset of radiographic knee osteoarthritis: data from the osteoarthritis initiative. Ann Rheum Dis. 2015; 74(7):1353-1359. [PubMed: 24615539]

10. Group KobotWHOS. Technical Report. University of Sheffield; UK: 2008. Assessment of osteoporosis at the primary health-care level.

11. Peterfy C, Schneider E, Nevitt M. The osteoarthritis initiative: report on the design rationale for the magnetic resonance imaging protocol for the knee. Osteoarthritis and Cartilage. 2008; 16:14331441. [PubMed: 18786841]

12. Ehrich EW, Davies GM, Watson DJ, Bolognese JA, Seidenberg BC, Bellamy N. Minimal perceptible clinical improvement with the Western Ontario and McMaster Universities osteoarthritis index questionnaire and global assessments in patients with osteoarthritis. The Journal of rheumatology. 2000; 27(11):2635-2641. [PubMed: 11093446]

13. Baum T, Stehling C, Joseph GB, et al. Changes in knee cartilage T2 values over 24 months in subjects with and without risk factors for knee osteoarthritis and their association with focal knee 
lesions at baseline: data from the osteoarthritis initiative. Journal of magnetic resonance imaging : JMRI. 2012; 35(2):370-378. [PubMed: 21987496]

14. Joseph GB, Baum T, Carballido-Gamio J, et al. Texture analysis of cartilage T2 maps: individuals with risk factors for OA have higher and more heterogeneous knee cartilage MR T2 compared to normal controls--data from the osteoarthritis initiative. Arthritis research \& therapy. 2011; 13(5):R153. [PubMed: 21933394]

15. Stehling C, Lane NE, Nevitt MC, Lynch J, McCulloch CE, Link TM. Subjects with higher physical activity levels have more severe focal knee lesions diagnosed with 3T MRI: analysis of a nonsymptomatic cohort of the osteoarthritis initiative. Osteoarthritis Cartilage. 2010; 18(6):776-786. [PubMed: 20202488]

16. Kretzschmar M, Lin W, Nardo L, et al. Association of Physical Activity Measured by Accelerometer, Knee Joint Abnormalities, and Cartilage T2 Measurements Obtained From 3T Magnetic Resonance Imaging: Data From the Osteoarthritis Initiative. Arthritis care \& research. 2015; 67(9):1272-1280. [PubMed: 25777255]

17. Gersing AS, Solka M, Joseph GB, et al. Progression of cartilage degeneration and clinical symptoms in obese and overweight individuals is dependent on the amount of weight loss: 48month data from the Osteoarthritis Initiative. Osteoarthritis Cartilage. 2016

18. Peterfy C, Schneider E, Nevitt M. The osteoarthritis initiative: report on the design rationale for the magnetic resonance imaging protocol for the knee. Osteoarthritis and cartilage/OARS, Osteoarthritis Research Society. 2008; 16(12):1433.

19. Kellgren J, Lawrence J. Radiologic assessment of osteoarthritis. Ann Rheum Dis. 1957; 16:494502. [PubMed: 13498604]

20. Iranpour-Boroujeni T, Li J, Lynch JA, Nevitt M, Duryea J. Investigators OAI. A new method to measure anatomic knee alignment for large studies of OA: data from the osteoarthritis initiative. Osteoarthritis Cartilage. 2014; 22(10):1668-1674. [PubMed: 25278076]

21. Peterfy CG, Guermazi A, Zaim S, et al. Whole-Organ Magnetic Resonance Imaging Score (WORMS) of the knee in osteoarthritis. Osteoarthritis Cartilage. 2004; 12(3):177-190. [PubMed: 14972335]

22. Stehling C, Baum T, Mueller-Hoecker C, et al. A novel fast knee cartilage segmentation technique for T2 measurements at MR imaging--data from the Osteoarthritis Initiative. Osteoarthritis Cartilage. 2011; 19(8):984-989. [PubMed: 21515391]

23. Joseph GB, Baum T, Alizai H, et al. Baseline mean and heterogeneity of MR cartilage T2 are associated with morphologic degeneration of cartilage, meniscus, and bone marrow over 3 years-data from the Osteoarthritis Initiative. Osteoarthritis and cartilage. 2012; 20(7):727-735. [PubMed: 22503812]

24. Miller AJ, Joseph PM. The use of power images to perform quantitative analysis on low SNR MR images. Magn Reson Imaging. 1993; 11(7):1051-1056. [PubMed: 8231670]

25. Raya J, Dietrich O, Horng A, Weber J, Reiser M, Glaser C. T2 measurement in articular cartilage: Impact of the fitting method on accuracy and precision at low SNR. Magnetic Resonance in Medicine. 2010; 63(1):181-193. [PubMed: 19859960]

26. Braun HJ, Gold GE. Diagnosis of osteoarthritis: imaging. Bone. 2012; 51(2):278-288. [PubMed: 22155587]

27. Lindsey C, Sheather S. Best subsets variable selection in nonnormal regression models. The Stata Journal. 2015; 14(4):1046-1059.

28. Furnival GM, Wilson RW. Regressions by Leaps and Bounds. Technometrics. 1974; 16:499-511.

29. Burnham, KP., Anderson, DR. Model Selection and Multi-Model Inference: A Practical Information-Theoretic Approach. 2002.

30. Pencina MJ, D’Agostino RB Sr, Steyerberg EW. Extensions of net reclassification improvement calculations to measure usefulness of new biomarkers. Statistics in medicine. 2011; 30(1):11-21. [PubMed: 21204120]

31. Refaeilzadeh, P., Tang, L., Liu, H. Encyclopedia of database systems. Springer; 2009. Crossvalidation; p. 532-538. 
32. DeLong ER, DeLong DM, Clarke-Pearson DL. Comparing the areas under two or more correlated receiver operating characteristic curves: a nonparametric approach. Biometrics. 1988; 44(3):837845. [PubMed: 3203132]

33. Joseph GB, McCulloch CE, Nevitt MC, et al. A reference database of cartilage 3 T MRI T2 values in knees without diagnostic evidence of cartilage degeneration: data from the osteoarthritis initiative. Osteoarthritis Cartilage. 2015; 23(6):897-905. [PubMed: 25680652]

34. Losina E, Klara K, Michl GL, Collins JE, Katz JN. Development and feasibility of a personalized, interactive risk calculator for knee osteoarthritis. BMC musculoskeletal disorders. 2015; 16:312. [PubMed: 26494421]

35. Kerkhof HJ, Bierma-Zeinstra SM, Arden NK, et al. Prediction model for knee osteoarthritis incidence, including clinical, genetic and biochemical risk factors. Ann Rheum Dis. 2014; 73(12): 2116-2121. [PubMed: 23962456]

36. Zhang W, McWilliams DF, Ingham SL, et al. Nottingham knee osteoarthritis risk prediction models. Ann Rheum Dis. 2011; 70(9):1599-1604. [PubMed: 21613308]

37. Yoo TK, Kim DW, Choi SB, Oh E, Park JS. Simple Scoring System and Artificial Neural Network for Knee Osteoarthritis Risk Prediction: A Cross-Sectional Study. PloS one. 2016; 11(2):e0148724. [PubMed: 26859664]

38. Urish, K., Keffalas, M., Durkin, J., Miller, D., Chu, C., Mosher, T. Signal Heterogeneity on Cartilage T2 Maps Predict Rapid Symptomatic Progression of OA. ORS; San Francisco, CA: 2012.

39. Joseph GB, Baum T, Alizai H, et al. Baseline mean and heterogeneity of MR cartilage T2 are associated with morphologic degeneration of cartilage, meniscus, and bone marrow over 3 years-data from the Osteoarthritis Initiative. Osteoarthritis Cartilage. 2012; 20(7):727-735. [PubMed: 22503812]

40. Strover, S. Understanding arthritis - compartments. Associates Ltd; 2008. Understanding Arthritis of the Knee.

41. Takatalo J, Karppinen J, Niinimaki J, et al. Prevalence of degenerative imaging findings in lumbar magnetic resonance imaging among young adults. Spine (Phila Pa 1976). 2009; 34(16):17161721. [PubMed: 19770614]

42. Eckstein F, Maschek S, Wirth W, et al. One year change of knee cartilage morphology in the first release of participants from the Osteoarthritis Initiative progression subcohort: association with sex, body mass index, symptoms and radiographic osteoarthritis status. Ann Rheum Dis. 2009; 68(5):674-679. [PubMed: 18519425]

43. Bucknor MD, Nardo L, Joseph GB, et al. Association of cartilage degeneration with four year weight gain--3T MRI data from the Osteoarthritis Initiative. Osteoarthritis Cartilage. 2015; 23(4): 525-531. [PubMed: 25591445]

44. Lin W, Alizai H, Joseph GB, et al. Physical activity in relation to knee cartilage T2 progression measured with 3 T MRI over a period of 4 years: data from the Osteoarthritis Initiative. Osteoarthritis Cartilage. 2013; 21(10):1558-1566. [PubMed: 23831632]

45. Runhaar J, de Vos BC, van Middelkoop M, Vroegindeweij D, Oei EH, Bierma-Zeinstra SM. Moderate weight loss prevents incident knee osteoarthritis in overweight and obese females. Arthritis care \& research. 2016

46. Roos EM, Dahlberg L. Positive effects of moderate exercise on glycosaminoglycan content in knee cartilage: a four-month, randomized, controlled trial in patients at risk of osteoarthritis. Arthritis Rheum. 2005; 52(11):3507-3514. [PubMed: 16258919]

47. Johns DJ, Hartmann-Boyce J, Jebb SA, Aveyard P. Behavioural Weight Management Review G. Diet or exercise interventions vs combined behavioral weight management programs: a systematic review and meta-analysis of direct comparisons. Journal of the Academy of Nutrition and Dietetics. 2014; 114(10):1557-1568. [PubMed: 25257365]

48. Teichtahl AJ, Wluka AE, Tanamas SK, et al. Weight change and change in tibial cartilage volume and symptoms in obese adults. Ann Rheum Dis. 2015; 74(6):1024-1029. [PubMed: 24519241]

49. Anandacoomarasamy A, Leibman S, Smith G, et al. Weight loss in obese people has structuremodifying effects on medial but not on lateral knee articular cartilage. Ann Rheum Dis. 2012; 71(1):26-32. [PubMed: 22135412] 
50. LaBella CR, Huxford MR, Grissom J, Kim KY, Peng J, Christoffel KK. Effect of neuromuscular warm-up on injuries in female soccer and basketball athletes in urban public high schools: cluster randomized controlled trial. Archives of pediatrics \& adolescent medicine. 2011; 165(11):10331040. [PubMed: 22065184]

51. Hewett TE, Lindenfeld TN, Riccobene JV, Noyes FR. The effect of neuromuscular training on the incidence of knee injury in female athletes. A prospective study. Am J Sports Med. 1999; 27(6): 699-706. [PubMed: 10569353]

52. WHO. Technical Report: Assessment of Fracture Risk and its Application to Screening for Postmenopausal Osteoporosis: a report of a WHO study group. World Health Organization; Geneva, Switzerland: 1994. Series 843

53. Fripp J, Crozier S, Warfield SK, Ourselin S. Automatic segmentation and quantitative analysis of the articular cartilages from magnetic resonance images of the knee. IEEE Trans Med Imaging. 2010; 29(1):55-64. [PubMed: 19520633]

54. Pedoia V, Li X, Su F, Calixto N, Majumdar S. Fully automatic analysis of the knee articular cartilage T1rho relaxation time using voxel-based relaxometry. J Magn Reson Imaging. 2016; 43(4):970-980. [PubMed: 26443990]

55. Gersing AS, Schwaiger BJ, Heilmeier U, et al. Evaluation of Chondrocalcinosis and Associated Knee Joint Degeneration Using MR Imaging: Data from the Osteoarthritis Initiative. Eur Radiol. 2017; 27(6):2497-2506. [PubMed: 27704199] 


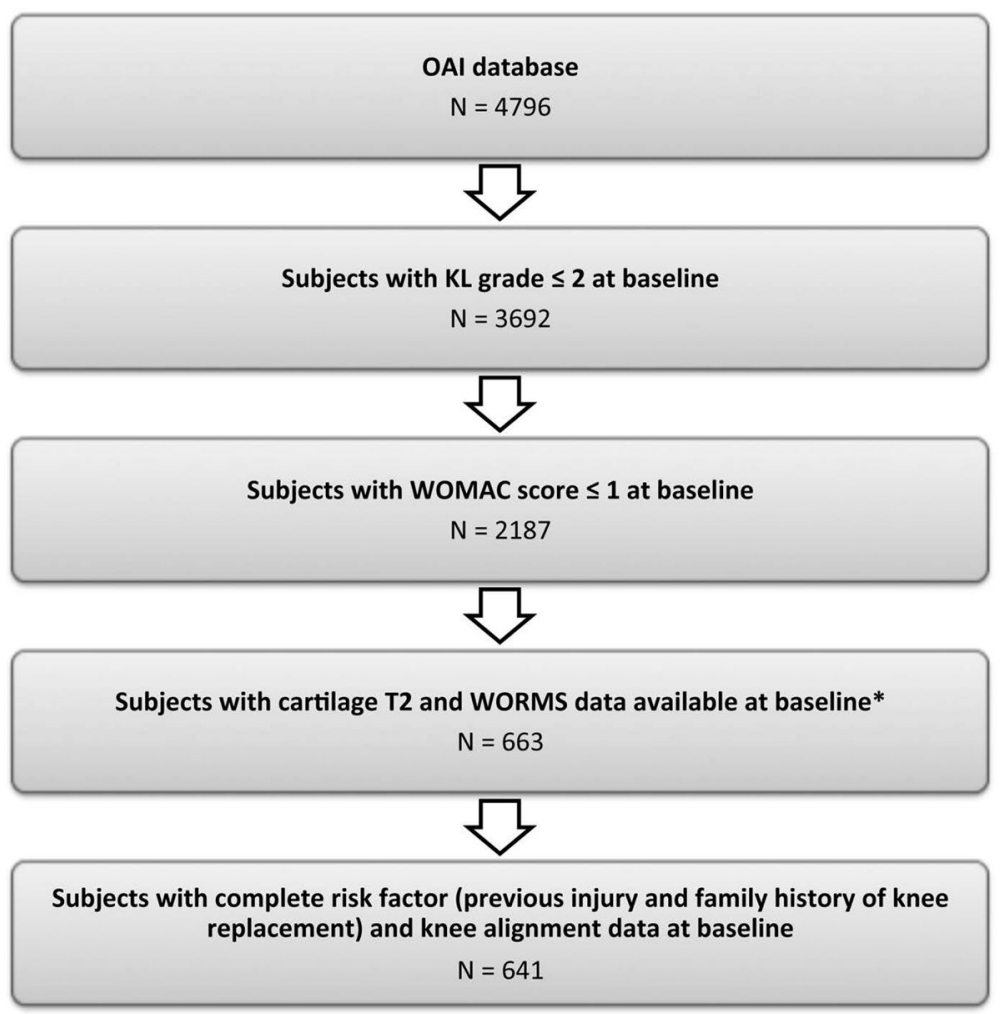

Figure 1.

Subject selection flowchart. Subjects were selected based on measurements in the right knee. * The sample of knees was selected from previous analyses of T2 measurements and WORMS scores (13-17). 


\begin{tabular}{|c|c|c|c|}
\hline & \multicolumn{3}{|c|}{$\begin{array}{l}\text { Subjects without symptomatic or radiographic } \mathrm{OA} \\
\qquad N=641\end{array}$} \\
\hline & \& & \ & 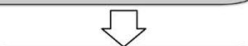 \\
\hline & Model 1: & Model 2: & Model 3: \\
\hline & Risk Factors + + -ray & $\begin{array}{l}\text { Risk Factors + X-ray } \\
+ \text { MRI WORMS }\end{array}$ & $\begin{array}{l}\text { Risk Factors + X-ray + MRI } \\
\text { WORMS + MRI cartilage T2 }\end{array}$ \\
\hline \multicolumn{4}{|l|}{ Subject Demographics } \\
\hline Age, gender, BMI & $\mathrm{x}$ & $\mathrm{x}$ & $\mathrm{x}$ \\
\hline \multicolumn{4}{|l|}{ Risk Factors: } \\
\hline Knee Injury ever & $x$ & $x$ & $x$ \\
\hline Knee Injury in last 12 months & $\mathrm{x}$ & $\mathrm{x}$ & $\mathrm{x}$ \\
\hline Fam. History of TKR & $x$ & $\mathrm{x}$ & $\mathrm{x}$ \\
\hline \multicolumn{4}{|l|}{$\underline{\mathrm{x} \text {-ray: }}$} \\
\hline KL Score & $\mathrm{x}$ & $\mathrm{x}$ & $x$ \\
\hline Alignment Angle & $x$ & $\mathrm{x}$ & $x$ \\
\hline \multicolumn{4}{|l|}{ MRI WORMS: } \\
\hline $\begin{array}{l}\text { Cartilage defect: MF, MT, LF, LT, } \\
\text { PAT, any }\end{array}$ & & $\mathrm{x}$ & $\mathrm{x}$ \\
\hline $\begin{array}{l}\text { Meniscus Tear: Med Ant, Body, } \\
\text { Post; Lat Ant, Body, Post; Any }\end{array}$ & & $\mathrm{x}$ & $\mathrm{x}$ \\
\hline \multicolumn{4}{|l|}{ MRI Cartilage T2: } \\
\hline \multirow[t]{5}{*}{ MF, MT, LF, LT, PAT, Mean. } & & & $\mathrm{x}$ \\
\hline & \multicolumn{3}{|c|}{ そ } \\
\hline & \multicolumn{3}{|c|}{$\begin{array}{l}\text { Model Building } \\
\text { Using STATA's all possible subsets algorithm } \rightarrow \text { Lowest AIC }\end{array}$} \\
\hline & \multicolumn{3}{|c|}{$\sqrt{3}$} \\
\hline & \multicolumn{3}{|c|}{$\begin{array}{l}\text { 10-fold Cross Validation } \\
\text { Highest Discrimination Index }\end{array}$} \\
\hline
\end{tabular}

Figure 2.

Illustration of the development and validation of three risk prediction models. 


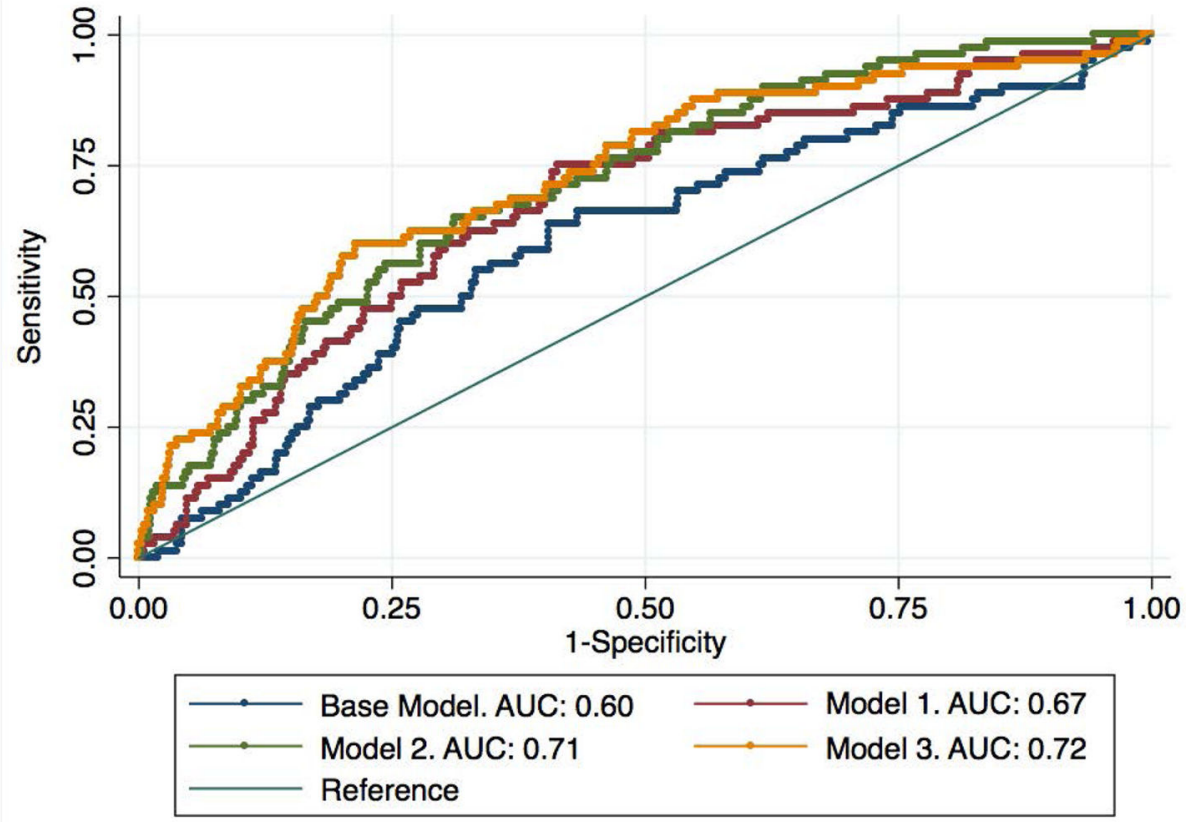

Figure 3.

Illustration of the ROC curves for the risk prediction models. The Base Model includes Age, gender, and BMI. Model $1=$ Base + X-ray + previous injury (12 months); Model $2=$ Model $1+$ WORMS; Model 3 = Model $2+$ T2 . 


\section{Risk Probability Subdivided by Progression}

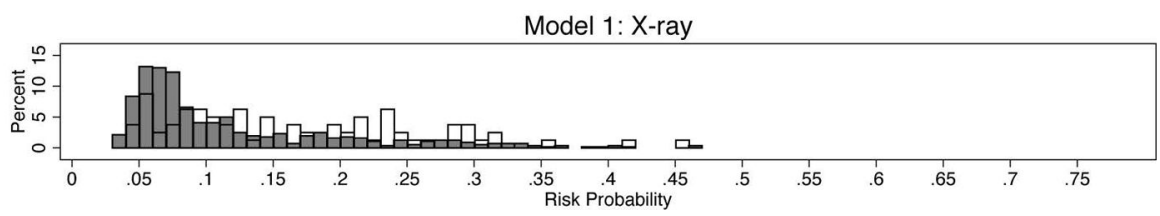

Model 2: X-ray + WORMS

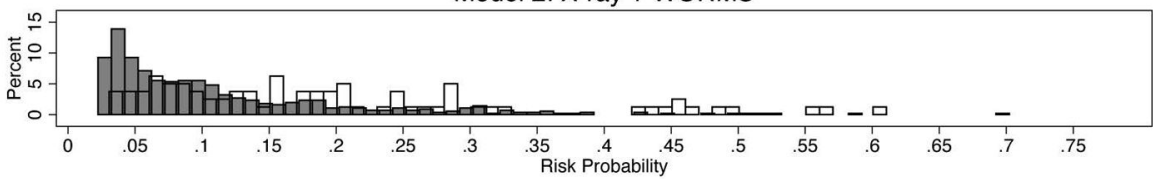

Model 3: X-ray + WORMS + Cartilage T2

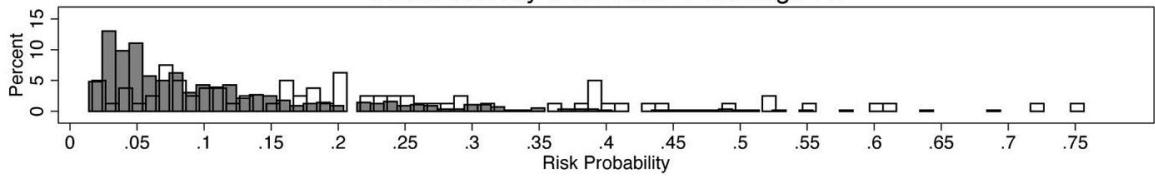

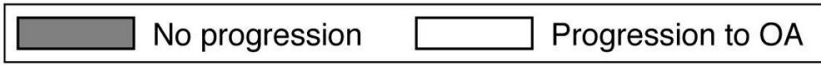

Figure 4.

The model classification improves from Model 1 (radiography + Risk factors) to Model 3 (Model $1+$ WORMS + T2), as shown by the increasing spread of the data. The higher the risk probability, the higher the likelihood for progression - this phenomenon is especially pronounced when comparing Models 1 to Model 3. 

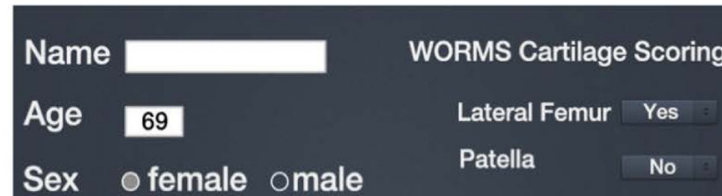

Weight

$150 \mathrm{lb}$

Height

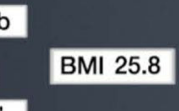

Mean T2 Values

5' 4"

Medial Tibia

Medial Femur

30.9

Kellgren \& Lawrence Grading

Meniscal tear present

Previous injung in the last $12 \mathrm{~m}$

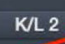

Yes

Yes

Risk probability

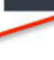

Medial Femur T2 [ms] 30.8

Medial Femur T2 [ms]

37.7

Medial Femur T2 [ms]

42.3
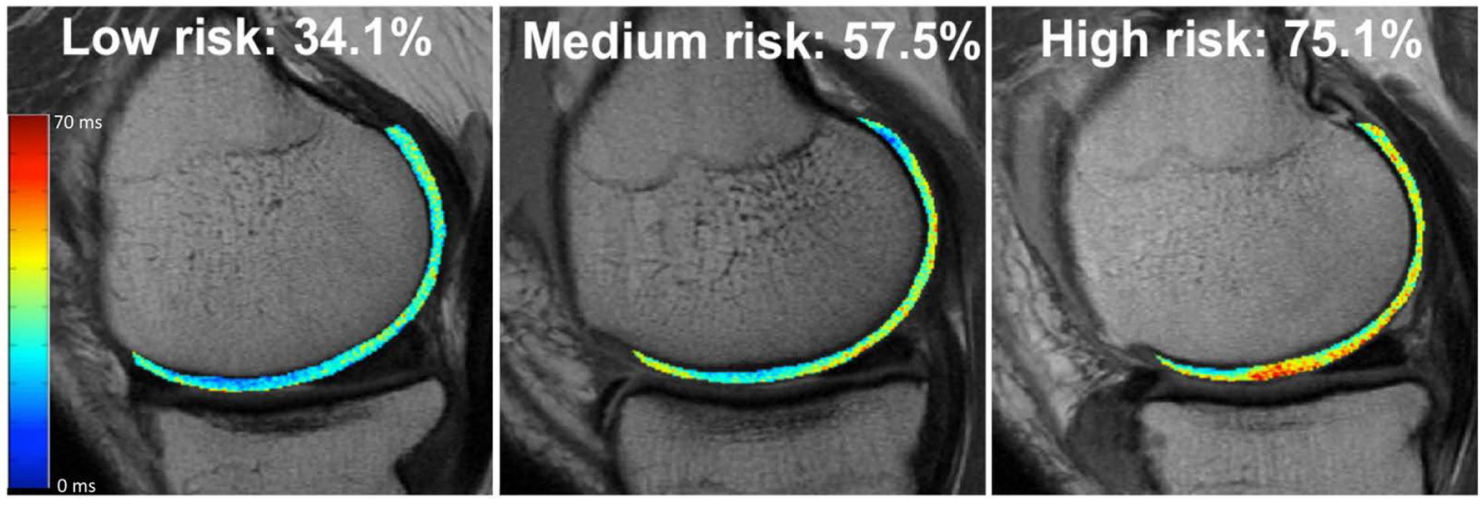

Figure 5.

(a) A graphic of the Risk Score calculator, (b) An illustration of the effects of cartilage T2 on OA risk prediction, while keeping the subject characteristics including KL and WORMS scores constant. As cartilage T2 increases, the risk for OA development increases, as illustrated by the red areas in the "high risk" T2 map. 


\section{Table 1}

Participant Characteristics.

\begin{tabular}{ll}
\hline & All Participants \\
\hline $\mathbf{n}$ & 641 \\
\hline Age (years) & $56.38 \pm 7.47$ \\
\hline BMI $\left(\mathbf{k g} / \mathbf{m}^{\mathbf{2}}\right)$ & $27.02 \pm 4.29$ \\
\hline Gender (male) & $358(55.85 \%)$ \\
\hline WOMAC ${ }^{*}$ pain & $0.17 \pm 0.38$ \\
\hline Family history of knee replacement & $81(12.64 \%)$ \\
\hline Previous injury anytime & $140(21.68 \%)$ \\
\hline Previous injury in the last 12 months & $12(1.87 \%)$ \\
\hline KL & \\
\hline $\mathbf{0}$ & $398(62.09 \%)$ \\
\hline $\mathbf{1}$ & $121(18.80 \%)$ \\
\hline $\mathbf{2}$ & $122(19.03 \%)$ \\
\hline
\end{tabular}

WOMAC: Western Ontario and McMaster Universities 
Joseph et al.

Page 20

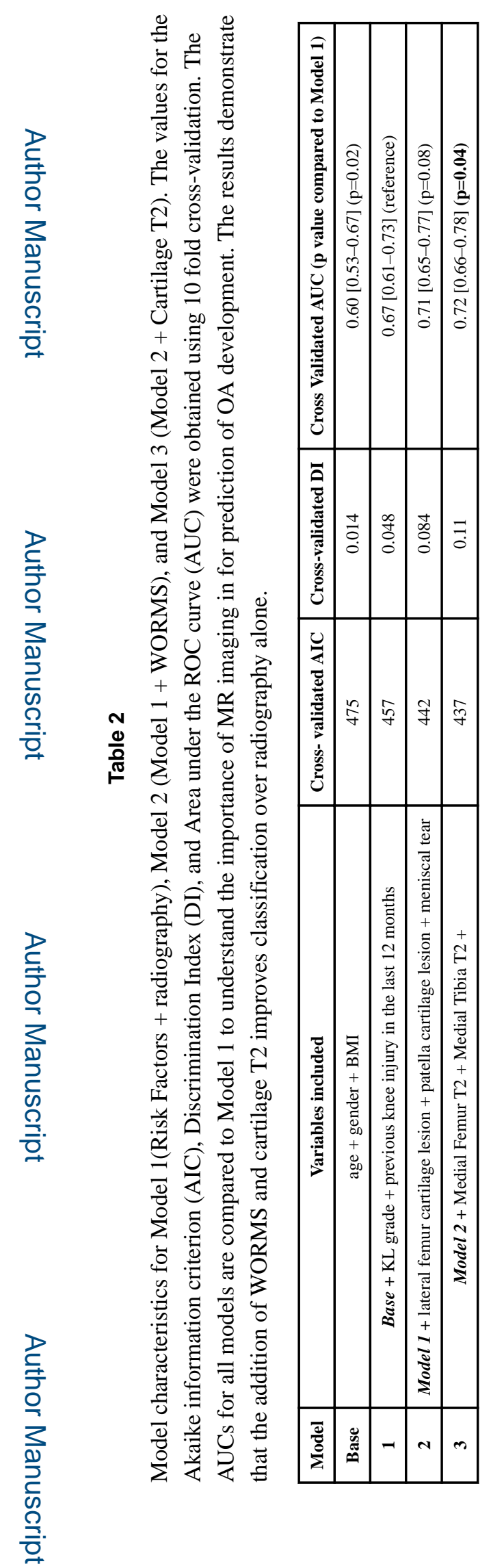

J Magn Reson Imaging. Author manuscript; available in PMC 2019 June 01. 